\title{
BOTULINUM INJECTION IN THE MASTICATORY MUSCLES FOR MANAGEMENT OF MYOFASCIAL PAIN
}

\author{
Ahmad Ibrahim Nagy Fathy ${ }^{1}$, Khaled Mohmmad Ali $^{2}$, Ahmad Mohammad Mostafa $^{3}$
}

\begin{abstract}
Objective: The objective of this study was to evaluate the effectiveness of botulinum toxin injection in the masseter and temporalis muscles compared to hard maxillary occlusal splint in management of myofascial pain.

Subjects and Methods: This study was done on forty adult patients suffering from myofascial pain, divided into two groups; study group (patients were injected with botulinum toxin A in masseter and temporalis muscles bilaterally), and control group (patients were managed by wearing a hard maxillary occlusal splint). The patients were evaluated by electromyogram EMG, Visual analogue scale VAS, muscle tenderness, and maximum mouth opening (pain free).

Results: Control group had a higher significant value of EMG than study group after three months. Control group had a higher significant value of VAS than study group after two, three and four weeks, and after two, three and four months. Study group had a higher significant value of VAS than control group after one week and six months. Control group had a higher significant value of muscle tenderness than study group after one and three months, while study group had a higher significant value of muscle tenderness than control group after six months. Study group had a higher significant value of maximum mouth opening (pain free) after two, three and four months. Control group had a higher significant value of maximum mouth opening (pain free) after one week and six months.
\end{abstract}

Conclusion: Bilateral injections of botulinum toxin in masseter and temporalis muscles was a successful treatment for myofascial pain.

KEYWORDS: Botulinum, masticatory muscles, myofascial pain, occlusal splint.

\section{INTRODUCTION}

Temporomandibular diseases (TMDs) is a collective term used to describe a number of related disorders affecting temporomandibular joints (TMJs), masticatory muscles and associated structures, all of which have common signs and symptoms that include complaints of facial and TMJ pain, tenderness to palpation of face and TMJ, uncoordinated mandibular movements and the presence of joint sounds (1). Myofascial pain syndrome (MPS) is defined as a regional pain disorder of muscular origin characterized by the existence of trigger points within muscles ${ }^{(2)}$. The myofascial trigger point (MTrP) is, in turn, defined as a palpable and hyperirritable nodule located in a taut band of muscle. Stimulation

\footnotetext{
* Master Candidate, Dentist at Ministry of Health.

** Professor, Department of Oral and Maxillofacial Surgery, Faculty of Dental Medicine (Boys - Cairo), Al-Azhar University

*** Lecturer of Department of Oral and Maxillofacial Surgery. Faculty of Dental Medicine, (Boys - Cairo), Al-Azhar University
}

-Corresponding author: ahmednagy131087@gmail.com

DOI: 10.21608 /ajdsm.2020.25075.1013 
of this point produces two characteristic phenomena: referred pain, and sudden contractions of the taut band, which is called the local twitch response (LTR). Usual treatment of temporomandibular myofascial pain is a combination of pharmacological and splint therapy, which produces a temporary relief of painful symptoms. However, pharmacological treatments soon reach the limit of therapeutic efficacy and they are also associated with side effects (gastrointestinal disorders, drug interactions and adverse reactions), so that the current trend is the search for alternative treatments ${ }^{(3)}$.

Botulinum toxin (BTX/Botox) is a potent bacterial neurotoxin produced by the anaerobic bacterium Clostridium botulinum. Botulinum toxin acts by blocking neuromuscular conduction and produces muscle paralysis, atrophy and weakness. The toxin has been extensively used for the control of involuntary muscle spasm and for cosmetic treatment ${ }^{(4)}$.
Botulinum toxin has also been used for the treatment of hyperfunctional muscles or muscle spasm ${ }^{(5,6)}$.

Hence, the purpose of this study was to evaluate the effectiveness of intramuscular injections of botulinum toxin in management of patients with myofascial pain compared to conservative treatment (occlusal splint).

\section{SUBJECTS AND METHODS}

This study was done on forty adult patients suffering from myofascial pain, divided into two groups. In the study group patients were injected with botulinum toxin $\mathrm{A}$ in masseter and temporalis muscles bilaterally, and in the control group they were managed by wearing a hard maxillary occlusal splint. The patients were evaluated by electromyogram EMG, Visual analogue scale VAS, evaluation of muscle tenderness, and maximum mouth opening (pain free).

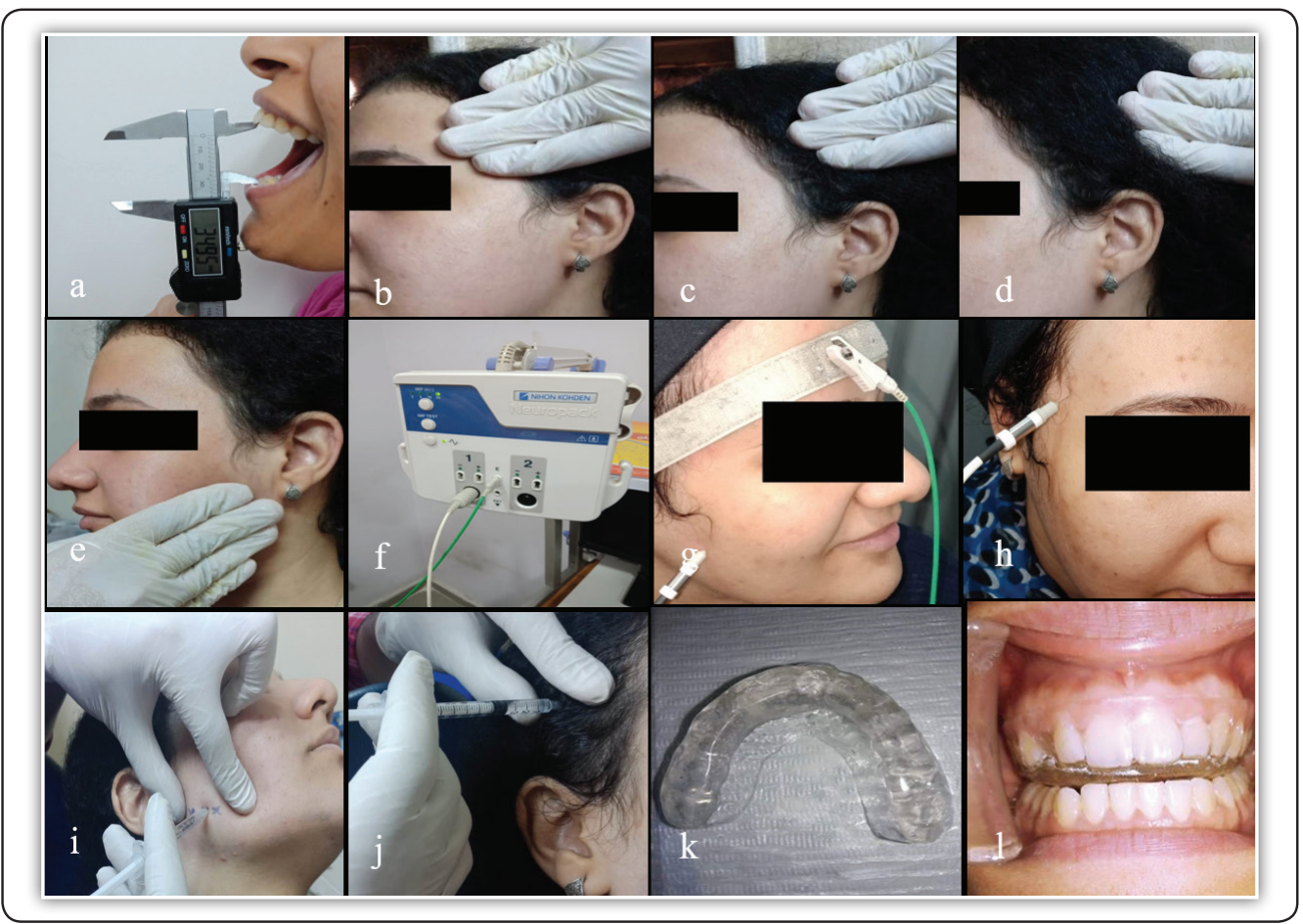

FIG (1) a; Measurement of maximum interincisal mouth opening (pain free) using vernier digital electronic caliper, $\mathbf{b}$; Palpation of anterior vertical fibers of temporalis muscle, $\mathbf{c}$; Palpation of middle oblique fibers of temporalis muscle, $\mathbf{d}$; Palpation of posterior horizontal fibers of temporalis muscle e; Palpation of masseter muscle, $\mathbf{f}$; Electromyogram device, $\mathbf{g}$; Needle EMG of the masseter muscle, $\mathbf{h}$, Needle EMG of the temporalis muscle ,i, Injection of masseter muscle with botulinum toxin, $\mathbf{j}$, Injection of temporalis muscle with botulinum toxin, k; Maxillary occlusal splint, l; Frontal view showing maxillary occlusal splint . 
Patients fulfilled the following criteria were included in this study: Age: 20-40 years old, suffering from chronic myofascial pain, limited jaw movement, having headache, and no physical evidence of internal derangement either with or without reduction.

Any patient with one of the following conditions was excluded from this study; Allergy to BTX-A (determined by skin test), active inflammation or infection at the proposed injection site, chronic degenerative neuromuscular disorders, history of any treatment for bruxism and/or TMD during six months prior to the study, previous surgery in TMJ and muscles, osteoarthritis, TMJ dislocation, internal derangement, and pregnancy and lactation.

\section{Sample size calculation}

According to analyses of the pain score, sample size calculation was undertaken via $G$ power version 3.1 statistical software using $(4.2 \pm 2.9$ for group I and $1.9 \pm 0.97$ for group II) : an alpha-type error of 0.05 , a power test of 0.95 , with Effect size used in calculation: $\mathrm{d}=1.0636926$. a total sample of at least 40 subjects 20 subjects for each group) appeared to be sufficient.

\section{RESULTS}

EMG recordings of the masseter and temporalis muscles obtained from study group patients injected with Botox, after three months of injection, showed a significant decrease in muscles amplitude especially muscles with high amplitude. And after six months, EMG readings elevated to the level of readings before injection or slightly less. And in control group patients who wore occlusal splints, they show no significant change in muscles amplitude over months. Control group had a higher significant value of EMG than study group after three months.

VAS obtained from study group patients injected with Botox showed a significant decrease after two, three and four weeks, and after two, three, and four months, and started to increase significantly after five months to reach nearly the original value or slightly less after six months. But in control group patients who wore occlusal splints, showed significant decreases, but less than those of Botox group till four weeks, then values remained constant till reaching six months.

Control group had a higher significant value of VAS than study group after two, three and four weeks, and after two, three and four months.

Study group had a higher significant value of VAS than control group after one week and six months.

Regarding muscle tenderness of temporalis and masseter muscles in study group patients injected with Botox, the level of tenderness showed a significant decrease after one and three months, and showed a significant increase to reach nearly the original value or slightly less after six months of injection. In control group patients who wore occlusal splints, the level of tenderness in masseter and temporalis muscles showed a significant decrease after one and three months, and remained constant after six months. Control group had a higher significant value of muscle tenderness than study group after one and three months, while study group had a higher significant value of muscle tenderness than control group after six months.

Regarding maximum mouth opening (pain free) calibration obtained from study group patients injected with Botox, it significantly increased after two weeks till reaching the fifth month when it started to decrease, but in control group patients who wore occlusal splints, it showed an insignificant increase. Study group had a higher significant value of maximum mouth opening (pain free) than control group after two, three and four months. Control group had a higher significant value of maximum mouth opening (pain free) than study group after one week and six months. 
TABLE (1): Comparison between the two studied groups according to mean maximum interincisal mouth opening, VAS, masseter muscle tenderness, and temporalis muscle tenderness.

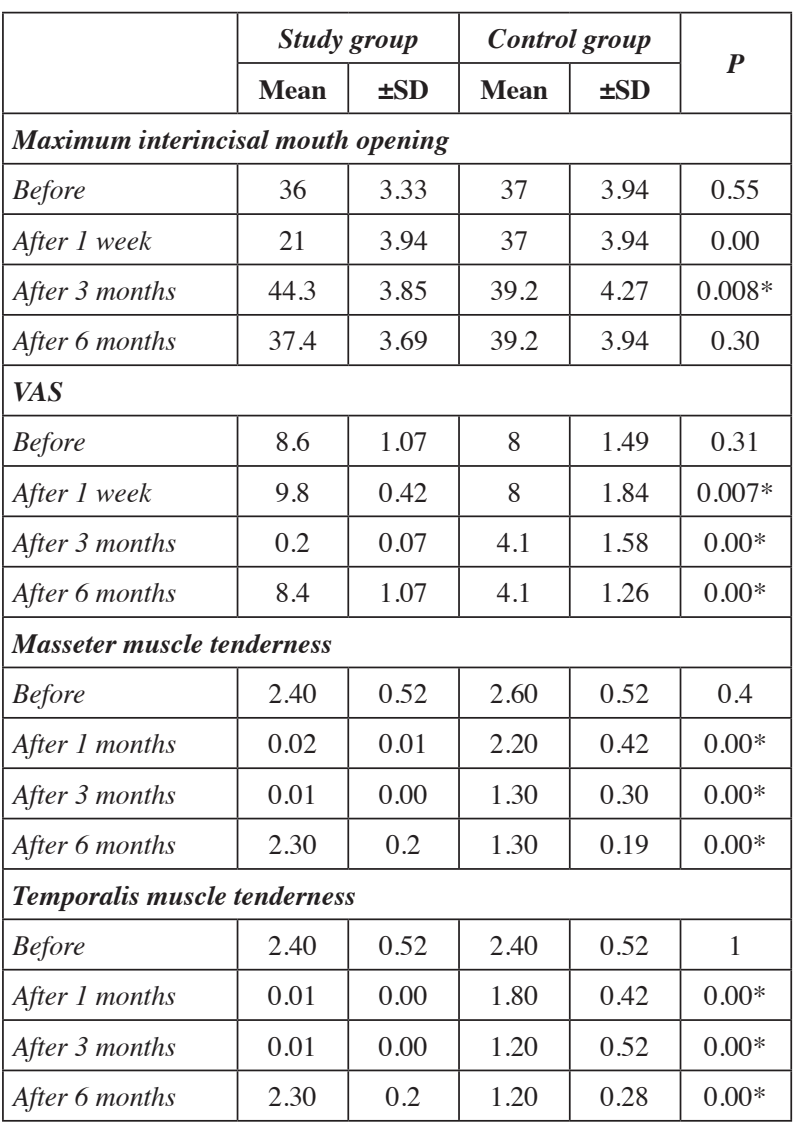

TABLE (2): Comparison between the two studied groups according to mean EMG amplitude $(\mu \mathrm{V})$ in masseter muscle and EMG amplitude $(\mu \mathrm{V})$ in temporalis muscle.

\begin{tabular}{|c|c|c|c|c|c|}
\hline & \multicolumn{2}{|c|}{ Study group } & \multicolumn{2}{|c|}{ Control group } & \multirow{2}{*}{$P$} \\
\hline & Mean & $\pm \mathrm{SD}$ & Mean & $\pm \mathrm{SD}$ & \\
\hline \multicolumn{6}{|c|}{ EMG amplitude $(\mu V)$ in masseter muscle } \\
\hline \multicolumn{6}{|l|}{ Left masseter } \\
\hline Before & 3235.75 & 586.36 & 3846.00 & 997.32 & 0.112 \\
\hline After 3 months & 1609.25 & 326.61 & 3843.33 & 666.65 & $0.000 *$ \\
\hline After 6 months & 3222.3 & 187.67 & 3865.4 & 572.4 & $0.0034^{*}$ \\
\hline \multicolumn{6}{|l|}{ Right masseter } \\
\hline Before & 3670.50 & 1200.61 & 3792.00 & 1326.26 & 0.832 \\
\hline After 3 months & 1862.75 & 1036.06 & 3656.67 & 1411.53 & $0.0045^{*}$ \\
\hline After 6 months & 3660.54 & 976.5 & 3821.6 & 1228.3 & 0.935 \\
\hline
\end{tabular}

\begin{tabular}{|c|c|c|c|c|c|}
\hline & \multicolumn{2}{|c|}{ Study group } & \multicolumn{2}{|c|}{ Control group } & \multirow{2}{*}{$\boldsymbol{P}$} \\
\hline & Mean & $\pm \mathrm{SD}$ & Mean & $\pm \mathrm{SD}$ & \\
\hline \multicolumn{6}{|c|}{ EMG amplitude $(\mu V)$ in temporalis muscle } \\
\hline \multicolumn{6}{|l|}{ Left masseter } \\
\hline Before & 3900.75 & 483.02 & 3396.33 & 574.35 & 0.466 \\
\hline After 3 months & 1949.50 & 155.38 & 3283.33 & 164.99 & $0.00 *$ \\
\hline After 6 months & 3389.6 & 417.3 & 3793.3 & 189.4 & $0.012 *$ \\
\hline \multicolumn{6}{|l|}{ Right masseter } \\
\hline Before & 2386.00 & 595.01 & 2335.67 & 319.76 & 0.816 \\
\hline After 3 months & 1376.00 & 201.52 & 2285.00 & 291.46 & $0.00 *$ \\
\hline After 6 months & 2327.2 & 518.4 & 2476.3 & 176.7 & 0.400 \\
\hline
\end{tabular}

$p: p$ value for comparing between the two studied groups *: Statistically significant at $p \leq 0.05$

\section{DISCUSSION}

In this study, preoperative electromyogram (EMG) recordings of the masseter and temporalis muscles on both sides were obtained from all patients in both groups. In each patient, most of muscles had a very high amplitude reading indicating muscle hyperactivity; this is in agreement with Shetty et al. ${ }^{(7)}$ that correlated bruxism and myofascial pain with muscle hyperactivity, and most of muscles had a high score of muscle tenderness (muscle pain) due to local muscle soreness, this is as that stated by Sinn et al ${ }^{(8)}$.

In study group, bilateral injections of botulinum toxin type $\mathrm{A}$ in both masseter and temporalis muscles reduced the frequency and severity of bruxism and myofascial pain in treated patients. These results are like the results of Monroy et al. ${ }^{(9)}$ who concluded that Botox effectively and safely improved bruxism and myofascial pain. They explained that, regardless its etiology, bruxism was mediated by contraction of the muscles that move the jaw. As botulinum toxin simply relaxes and weakness these muscles, so it is not surprising that it was beneficial.

Several studies emphasized that the effect of a single injection of botulinum toxin type A fades in period of around four months, which complies with the manufacturer's recommendations. In this study, 
at the end of the fourth-month follow up period, all patients started to regain their range of maximum mouth opening (pain free), and all muscles started regain their bite force after 4 months of botulinum toxin type A injection which goes in accordance with the results of Bakke et al ${ }^{(10)}$.

In this study, EMG recordings of masseter and temporalis had a significant decrease after 3 months, and then had a significant increase after 6 months. This agrees with Lee et al. ${ }^{(1)}$ who reported a decrease in EMG in case of bruxism when $80 \mathrm{U}$ of botulinum toxin were injected into the masseter muscle only for 3 months. In the other study done by Jankovic and $\operatorname{Tan}^{(12)}$, they demonstrated that injections of $62 \mathrm{U}$ of Botox in each masseter muscle could be a safe and effective treatment for severe tooth grinding for five months after injection. The previous studies concluded that injection of a small dose of botulinum toxin in masseter and temporalis muscles bilaterally might be more effective than injection of a large dose of Botox into the masseter muscle only. Grover et al. ${ }^{(13)}$ concluded that Botox injection in masseter and temporalis to be of the lowest possible dose to achieve the desired effect based on the size of masseter and temporalis muscle. Also, it is always possible to titrate up to a higher dose if necessary, so that Botox dose shouldn't be standardized to every patient.

The results of this study showed that there was a significant increase in maximum interincisal mouth opening (pain free).Results of the current study showed that the peak drop of maximum mouth opening (pain free) occurred within the first two weeks post-injection, and then started to increase from the third week which complies with the study performed by Kim et al ${ }^{(14)}$. This also complies with the results of earlier studies stating that the injected muscle's action dropped maximally within the first fourteen days, which was noted in the study performed by Kartoglu et al ${ }^{(15)}$.

The results of this study showed that there was a significant decrease in VAS and muscle tenderness.
This is in accordance with the study performed by Kartoglu et al. ${ }^{(15)}$ who stated that patients showed improvement in pain aspects after BTX-A injection in both masseter and temporalis muscles.

In this study, VAS increased one week after injection, and then decreased, this is due to local reactions to Botulinum toxin type $\mathrm{A}$ at the administration site which commonly include; pain, edema, erythema, ecchymosis, headache, and shortterm hyperesthesia ${ }^{(16)}$. In this study, VAS decreased two weeks after injection, this is as that shown in the results of Lindern et al. ${ }^{(17)}$, Fross ${ }^{(18)}$, who concluded that Botox injection decreased both VAS and muscle tenderness, and that facial and subjective pain decreased over time. Von Lindern et al. used BTX-A for eliminating local facial pain and reported an improvement in $91 \%$ of their subjects ${ }^{(17)}$. One of the reasons of decreased pain is the antinociceptive effect through blocking the release of inflammatory mediators, such as substance P and glutamate ${ }^{(19)}$.

In control group, the occlusal splint had side effects as patient incompliance with wearing an occlusal splint causing pain and sensitivity in teeth. This discomfort usually happens during the first few days of wearing the splint. The constant use can cause irritation of the patient's mouth and gum tissues, these results were the same results of Messing (20).

In this study, the use of hard maxillary occlusal splint did not affect muscles activity. This is in accordance with the results of Leonardo ${ }^{(21)}$ who aimed to evaluate long-term effects of using an occlusal splint in patients with bruxism and myofascial pain, using electromyography (EMG) of superficial masseter and anterior part of temporalis muscles, and concluded that there was no significant decrease in mean EMG levels over the therapy in the muscles. Also, Harada et al. ${ }^{(22)}$ study results showed that the occlusal splint reduced the masseter muscle activity during the night immediately after appliance installation. However, no effects were 
observed after 2, 4 and 6 weeks of use. The results of this study conflict with the results of Okeson ${ }^{(23)}$ who studied the effects of occlusal splint in the management of bruxism. He concluded that the use of the occlusal splint was associated with a reduction in motor activity of bruxism. Also, Clark et al. (24) showed that occlusal splint treatment resulted in a decrease in EMG activities, and Van der Zaag ${ }^{(25)}$ observed that, using occlusal splints decreased bruxism activity and EMG reading.

The results of this study reported that use of hard maxillary occlusal splint did not treat bruxism, but decreased significantly both VAS and muscle tenderness, and prevented teeth damage. The results agree with Macedo et al. ${ }^{(26)}$ who concluded that using of occlusal splints did not treat bruxism, but decreased VAS and prevented teeth damage. All interocclusal appliances temporarily increase the patient's vertical dimension. This effect is universal, regardless of treatment goals. It has been demonstrated that an increase in vertical dimension can be responsible for the symptom reduction ${ }^{(27)}$, Dao and Lavigne ${ }^{(28)}$ stated that; occlusal splints were proposed to treat bruxism, even though they were mainly directed to prevent bruxism-related damages (i.e., dental wear facets), rather than achieve a therapeutic effect. Holmgren et al. (29) and Harada et al. (22) concluded that the appliance did not cure bruxism; it only inhibited the bruxing tendency while it is being worn. And this explains the results of this study that showed that there was an insignificant increase in maximum mouth opening (pain free). These studies show that even after long term use of an appliance, bruxism returns if use of the device is stopped.

\section{CONCLUSION}

Bilateral injections of botulinum toxin type A (Botox) in both masseter and temporalis muscles were a successful treatment for myofascial pain dysfunction syndrome for six months.

\section{REFERENCES}

1. Conti PCR, Dos Santos CN, Koggawa EM, Conti ACF, De Araujo CRP: The treatment of painful temporomandibular joint clicking with oral splints: A randomized clinical trial. J Am Dent Assoc, 2006; 137:1108-14.

2. Jose M. Climent, Ta-Shen Kuan, Pedro Frenollosa and Fransisco Martin -del- Rosario: Botulinum toxin for the treatment of myofascial pain syndromes involving the nack\&back; A Review from a Clinical Perspective, 2013.

3. Simons DG: Review of enigmatic MTrPs as a common cause of enigmatic musculoskeletal pain and dysfunction. Journal of Electromyography and Kinesiology, 2004; 14(1):95-107.

4. Dolly JO, Aoki KR: The structure and mode of action of different botulinum toxins. European Journal of Neurology, 2006; 13(supplement 4):1-9.

5. Ernberg M, Hedenberg-Magnusson B, List T, Svensson P: Efficacy of botulinum toxin type A for treatment of persistent myofascial TMD pain: a randomized, controlled, double-blind multicenter study. Pain: 2011; 152(9):1988-96.

6. Mense $\mathrm{S}$ : Neurobiological basis for the use of botulinum toxin in pain therapy. Journal of Neurology, 2004; Supplement: 251(supplement 1):I1-I7.

7. Shetty S., Pitti V., Satish Babu C .L., Surendra Kumar G.P.\&Deepthi, B.C.: "Bruxism: a literature review", Journal of Indian Prosthodontic Society, 2010; 10 (3): 141-8.

8. Sinn D.P., de Assis E.A. \& Throckmorton G.S.:" Mandibular excursions and maximum bite forces in patients with temporomandibular joint disorders", J Oral Maxillofac Surg, 1996; 54:671-9.

9. Monroy P.G. \& da Fonseca M.A.:" The use of botulinum toxin-a in the treatment of severe bruxism in a patient with autism": a case report, Spec Care Dentist, Jan-Feb; 2006; 26(1):37-9.

10. Bakke M, Moller E, Werdelin L, Dalager T, Kitai N and kreiborg S. Treatment of severe temporomandibular joint clicking with botulinum toxin in the lateral pterygoid muscle in two cases of anterior disc displacement. Oral Surg Oral Med Oral Pathol Oral Radiol Endod, 2005; 100:693700 .

11. Lee, S., McCall, W., Kim\&Y. \& Chung, J..” Effect of botulinum toxin injection on nocturnal bruxism: A randomized controlled trial", American Journal of Medical Rehabilitation, 2010; 89:16-23.

12. Tan E.K. \& Jankovic J.:" Treating severe bruxism with botulinum toxin", J Am Dent Assoc, 2000; 131:211-6. 
13. Grover S, Malik V, Kaushik A,Diwakar R, Yadav P. A Future perspective of Botox in Dentofacial Region. J Pharm Biomed Sci; 2014; 4(6):525-31.

14. Kim K, Byun Y, Kim Y, Kim S. Muscle weakness after repeated injection of Botulinum toxin Type A, evaluated according to bite force measurement of human masseter muscle. Dermatol Surg, 2009; 35:1902-7.

15. Kurtoglu C, Gur O, Kurkcu, Sertdemir Y, Guler-Uysal F and Uysal H. Effect of Botulinum Toxin_A in myofscial pain dysfunction patients with or without functional disc displacement. J Oral Maxillofac Surg, 2008; 66:1644-51.

16. Girlanda P., Vita G., Nicolosi C., Milone S. \& Messina C.:" Botulinum toxin therapy: distant effects on neuromuscular transmission and autonomic nervous system", Journal of Neurology, Neurosurgery, and Psychiatry, 1992; 55: 844-5.

17. Von Lindern J.J, Niederhagen B., Berge S. \& Appel T.:'Type Abotulinum toxin in the treatment of chronic facial pain associated with masticatory hyperactivity", J. Oral Maxillofac. Surg, 2003; 6(7):774-8.

18. Fross R.D.:" Bruxism and masticatory myalgias: use of botulinum toxin", 2000; MOV, Visor,:15(suppl2):35.

19. Purkiss J., Welch M., Doward S. \& Foster K.” Capsaicinstimulated release of substance $\mathrm{P}$ from cultured dorsal root ganglion neurons: involvement of two distinct mechanisms", Biochem Pharmacol, 2000; 59:1403-6.

20. Messing S.G.:" Splint Therapy. In A. S. Kaplan \& L. A. Assael. Temporomandibular Disorders", Philadelphia: Saunders, 1992; 395-454.

21. Leonardo L.M.:" Occlusal splint for SB: an electromyographic associated to Helkimo Index evaluation", J of Pri- mary Care Sleep Medicine, 2004; 8: 275-82.

22. Harada T., Ichiki R., Tsukiyama Y. \& Koyano K.:" The effect of oral splint device son SB: a 6-week observation with an ambulatory electromyographic recording device", J Oral Rehabil, 2006; 33:482-8.

23. Okeson J.P.:" The effects of hard and soft occlusal splints on nocturnal bruxism", J Am Dent Assoc, 1987; 114:788-91.

24. Clark G.T., Beemsterboer P.L., Solberg W.K. \& Rugh J.D.: "Nocturnal electromyographic evaluation of myofascial pain dysfunction in patients undergoing occlusal splint therapy", J Am Dent Assoc, 1979; 99:607-11.

25. Van der Zaag J., Lobbezoo F., Wicks D.J., Visscher C.M., Hamburger H.L. \& Naeije M.:" Controlled assessment of the efficacy of occlusal stabilization splints on SB", journal of orofacial pain, 2005; 19 (2):151-8.

26. Macedo C.R., Silva A.B., Machado M.A., Saconato H. \& Prado G.F.: "Occlusal splints for treating SB (tooth grinding)", Cochrane Review In: The Cochrane Library Oxford; Issue 4, 2007.

27. Christensen L.V., Mohamed S.E. \& Harrison J.D.:" Delayed onset of masseter muscle pain in experimental tooth clenching", J Prosthet Dent, 1982; 48:579-84.

28. DaoT.T. \& Lavigne G.J.:" Oral splints: the crutches temporomandibular disorders and bruxism", Crit Rev Oral Biol Med, 1998; 9:345-61.

29. Holmgren K., Sheikholeslam A. \& Riise C.:" Effect of a full-arch maxillary occlusal splint on parafunctional activity during sleep in patients with nocturnal bruxism and signs and symptoms of craniomandibular disorders", J Prosthet Dent, 1993; 69:293-7. 